\title{
EXISTENCE AND NONEXISTENCE OF GLOBAL SOLUTIONS OF THE WAVE EQUATION WITH A NONLINEAR BOUNDARY CONDITION
}

\author{
$\mathrm{BY}$
}

\author{
AZMY S. ACKLEH AND KENG DENG
}

Department of Mathematics, University of Louisiana at Lafayette, Lafayette, Louisiana

Abstract. We study the initial-boundary value problem

$$
\begin{aligned}
& u_{t t}=u_{r x}, \quad 0<x<\infty, \quad t>0, \\
& -u_{r}(0, t)=h(u(0, t)), \quad t>0, \\
& u(x, 0)=f(x), \quad u_{t}(x, 0)=g(x), \quad 0<x<\infty .
\end{aligned}
$$

We establish criteria for existence and nonexistence of global solutions, and we present the growth rate at blow-up.

1. Introduction. In this paper, we consider the following initial-boundary value problem:

$$
\begin{aligned}
& u_{t t}=u_{x x}, \quad 0<x<\infty, \quad t>0, \\
& -u_{x}(0, t)=h(u(0, t)), \quad t>0, \\
& u(x, 0)=f(x), \quad u_{t}(x, 0)=g(x), \quad 0<x<\infty .
\end{aligned}
$$

Here, $h(u)$ and the initial values are continuous. The wave equation with similar boundary condition as in (1.1) arises in applied sciences. In order to motivate the main results for problem (1.1), we recall some old results for two related problems. On the one hand, several authors have studied the initial value problem

$$
\begin{array}{ll}
u_{t t}=u_{x x}+|u|^{p}, & -\infty<x<\infty, \quad t>0, \\
u(x, 0)=f(x), \quad u_{t}(x, 0)=g(x), & -\infty<x<\infty .
\end{array}
$$

Received April 26, 1999.

2000 Mathematics Subject Classification. Primary 35B40, 35L05, 35L15, 35L20.

Research of the first author was supported by the Louisiana Education Quality Support Fund under the grant LEQSF (1996-99)-RD-A-36. 
They showed that if $1<p<\infty$, every nontrivial solution of (1.2) blows up in finite time (see $[4,5,8])$. On the other hand, for the initial-boundary value problem

$$
\begin{array}{lll}
u_{t}=u_{x x}, & 0<x<\infty, & t>0, \\
-u_{x}(0, t)=u^{p}(0, t), & & t>0, \\
u(x, 0)=u_{0}(x), & 0<x<\infty, &
\end{array}
$$

it was shown [3] that if $1<p \leq 2$, all nonnegative solutions of (1.3) blow up in finite time while if $p>2$, there are nontrivial global solutions. Motivated by these results, in this paper we will establish criteria for global existence and finite time blow-up of solutions of problem (1.1) in Section 2. We will also present growth rates at blow-up for certain nonlinearities in Section 3.

2. Existence and nonexistence of global solutions. We begin this section with the definition of a solution of problem (1.1). Let $u(x, t)$ be a continuous function on $[0, \infty) \times[0, T)$ for some $T(0<T \leq \infty)$. Then $u$ is said to be a solution of $(1.1)$ if it satisfies the following: For $t \leq x$,

$$
u(x, t)=\frac{1}{2}[f(x+t)+f(x-t)]+\frac{1}{2} \int_{x-t}^{x+t} g(s) d s,
$$

and for $t>x$,

$u(x, t)=\frac{1}{2}[f(t+x)+f(t-x)]+\frac{1}{2}\left[\int_{0}^{t+x} g(s) d s+\int_{0}^{t-x} g(s) d s\right]+\int_{0}^{t-x} h(u(0, \tau)) d \tau$.

We first introduce the local existence of a solution of (1.1).

ThEOREM 2.1. There exists at least one solution of $(1.1)$ on $[0, \infty) \times\left[0, T_{0}\right)$ for some $T_{0}>0$. Moreover, if $h(u)$ is Lipschitz continuous, then the solution is unique.

Proof. In view of (2.1), it suffices to show that there is a function $u(x, t)$ satisfying

$$
u(0, t)=f(t)+\int_{0}^{t} g(s) d s+\int_{0}^{t} h(u(0, \tau)) d \tau
$$

The above equation is a Volterra integral equation. By Theorem 3.1.1 of [2], there exists at least one solution of $(2.2)$. Furthermore, if $h(u)$ is Lipschitz continuous, by Theorem 1.3.1 of [2], the solution is unique.

We then present the criteria for the global existence and finite time blow-up of solutions of (1.1).

ThEOREM 2.2. Suppose that $|h(u)| \leq p(|u|)$ with $\rho(r)>0$ continuous, nondecreasing on $[0, \infty)$, and such that

$$
\int^{\infty} \frac{d r}{\rho(r)}=\infty
$$

Then all solutions of (1.1) are global. 
Proof. Recalling Theorem 3.1.4 of [2] and setting $k(t, s, u)=h(u)$, one can see that $k$ satisfies the following condition:

$$
|k(t, s, u)| \leq \lambda(t) \rho(|u|)
$$

with $\lambda(t) \equiv 1$. Then by Remark 1, page 108 in [2], the result follows.

THEOREM 2.3. Suppose that $f(t)+\int_{0}^{t} g(s) d s \geq 0(\not \equiv 0)$ on $[0, \infty)$ and that $h(u) \geq \sigma(|u|)$ with $\sigma(r)>0$ continuous, nondecreasing on $[0, \infty)$, and such that

$$
\int^{\infty} \frac{d r}{\sigma(r)}<\infty
$$

Then every solution of (1.1) blows up in finite time.

Proof. Since $f(t)+\int_{0}^{t} g(s) d s \geq 0(\not \equiv 0)$, there is an interval $\left[t_{0}, t_{1}\right]$ over which $f(t)+$ $\int_{0}^{t} g(s) d s \geq d>0$. Then for $t_{0} \leq t \leq t_{1}, u(0, t) \geq d$ and $\int_{t_{0}}^{t_{1}} h(u(0, \tau)) d \tau \geq \sigma(d)\left(t_{1}-t_{0}\right)=$ $c_{0}>0$. Therefore for $t \geq t_{1}, u(0, t) \geq \int_{t_{0}}^{t} h(u(0, \tau)) d \tau \geq c_{0}$, and by comparison, $u(0, t) \geq \phi(t)$, where $\phi(t)$ satisfies

$$
\phi(t)=\frac{1}{2} c_{0}+\frac{1}{2} \int_{t_{0}}^{t} \sigma(\phi(\tau)) d \tau .
$$

Since $\int^{\infty} \frac{d r}{\sigma(r)}<\infty, \phi(t)$ must blow up in finite time, and so does $u(0, t)$.

For a certain class of nonlinearities, we can further weaken the condition on the initial data to show finite time blow-up.

THEOREM 2.4. Suppose that $\int_{0}^{\infty} f(t) d t+\int_{0}^{\infty} \int_{0}^{t} g(s) d s d t>0$ and $h(u) \geq c|u|^{p}(p>$ $1, c>0)$. Then the solution of (1.1) blows up in finite time.

Proof. Since $\int_{0}^{\infty} f(t) d t+\int_{0}^{\infty} \int_{0}^{t} g(s) d s d t>0$, for $t \geq \tilde{t}$ with sufficiently large $\tilde{t}$, $\int_{0}^{t} f(\tau) d \tau+\int_{0}^{t} \int_{0}^{\tau} g(s) d s d \tau \geq c_{1}>0$. Thus, by Jensen's inequality we have that for $t \geq 2 \tilde{t}$

$$
\begin{aligned}
\int_{0}^{t}|u(0, \tau)| d \tau & \geq c_{1}+c \int_{\tilde{t}}^{t} \int_{0}^{\tilde{t}}|u(0, s)|^{p} d s d \tau \\
& \geq c_{1}+c \int_{\tilde{t}}^{t} \tilde{t}^{1-p}\left(\int_{0}^{\tilde{t}}|u(0, s)| d s\right)^{p} d \tau \\
& \geq c_{1}+c \tilde{t}^{1-p} c_{1}^{p}(t-\tilde{t}) \\
& \geq c_{2} t
\end{aligned}
$$

where $c_{2}=c \tilde{t}^{1-p} c_{1}^{p} / 2$ 
We now choose a positive constant $\delta$ such that $1<\delta<\min \{2, p\}$. For $t \geq 2 \tilde{t}$, by $(2.3)$ we find

$$
\begin{aligned}
\int_{0}^{t}|u(0, \tau)| d \tau & \geq c_{1}+c \int_{2 i}^{t} \int_{0}^{\tau}|u(0, s)|^{p} d s d \tau \\
& \geq c_{1}+c \int_{2 i}^{t} \tau^{1-p}\left(\int_{0}^{\tau}|u(0, s)| d s\right)^{p} d \tau \\
& \geq c_{1}+c \int_{2 i}^{t} \tau^{1-p}\left(c_{2} \tau\right)^{p-\delta}\left(\int_{0}^{\tau}|u(0, s)| d s\right)^{\delta} d \tau .
\end{aligned}
$$

Thus by comparison, for $t \geq 2 \tilde{t}, \int_{0}^{t}|u(0, \tau)| d \tau \geq \psi(t)$, where $\psi(t)$ satisfies

$$
\begin{aligned}
\psi^{\prime}(t) & =c_{3} t^{1-\delta} \psi^{\delta}(t), \\
\psi(2 \tilde{t}) & =c_{1}
\end{aligned}
$$

with $c_{3}=c c_{2}^{p-\delta}$. Integration of $(2.4)$ over $(2 \tilde{t}, t)$ then yields

$$
\frac{c_{1}^{1-\delta}}{\delta-1}=\int_{c_{1}}^{\infty} \frac{d z}{z^{\delta}} \geq \int_{c_{1}}^{\iota \cdot(t)} \frac{d z}{z^{\delta}}=\frac{c_{3}}{2-\delta}\left(t^{2-\delta}-(2 \tilde{t})^{2-\delta}\right),
$$

which shows that $\psi(t)$ cannot exist globally. Therefore, $\int_{0}^{t}|u(0, \tau)| d \tau$, and hence $u(0, t)$ must blow up in finite time.

REMARK. Taking note of (2.1b), clearly, blow-up can occur only on the boundary.

3. Growth rate at blow-up. In this section, we are limiting ourselves to the situation where blow-up does occur in finite time, that is,

$$
u(0, t) \rightarrow \infty \quad \text { as } t \rightarrow \hat{t}<\infty .
$$

Based on this assumption, we will develop a self-consistent asymptotic analysis of (2.2). This asymptotic technique will describe the blow-up behavior of the solution. Our arguments parallel those of $[6,7]$, and therefore we only present some technical differences. Under the assumption (3.1), we introduce the transformation

$$
\eta=(\hat{t}-t)^{-1}-\eta_{0}, \quad \eta_{0}=(\hat{t})^{-1}, \quad w(\eta)=u(0, t) .
$$

Thus, (3.1) is equivalent to

$$
w(\eta) \rightarrow \infty \quad \text { as } \eta \rightarrow \infty .
$$

The transformation (3.2) converts (2.2) to the form

$$
w(\eta)=k(\eta)+\int_{0}^{\prime \prime} H(\zeta) d \zeta, \quad \eta \geq 0,
$$

where $k(\eta)=f(t)+\int_{0}^{t} g(s) d s$ and $H(\zeta)=\left(\zeta+\eta_{0}\right)^{-2} h(w(\zeta))$.

The advantage of this transformation is that certain techniques developed in [1] can be utilized for the asymptotic evaluation, as $\eta \rightarrow \infty$, of integrals like that in (3.4).

Following the methods of [1], let $\zeta=\eta \xi$, so that (3.4) becomes

$$
w(\eta)=k(\eta)+\eta \int_{0}^{1} H(\eta \xi) d \xi
$$


To investigate the asymptotic behavior of $w(\eta)$ as $\eta \rightarrow \infty$, the technique of [1] suggests employment of the Parseval formula for Mellin transforms to change the integral in (3.5) to one defined in the complex plane. Thus, as $\eta \rightarrow \infty,(3.5)$ is equivalent to

$$
w(\eta) \sim \frac{\eta}{2 \pi i} \int_{b-i x}^{b+i x} \frac{\eta^{-z}}{1-z} M[H(\xi) ; z] d z, \quad \eta \rightarrow \infty,
$$

where the Mellin transform is given by

$$
M[H(\xi) ; z] \equiv \int_{0}^{\infty} \xi^{z-1} H(\xi) d \xi .
$$

In order to carry out the asymptotic analysis of (3.6), we need more information about $M[H ; z]$. To this end, we first let $h(w) \sim w^{p}(p>1)$ and assume that

$$
w(\eta) \sim A \eta^{\lambda} \quad \text { as } \eta \rightarrow \infty,
$$

where $A$ and $\lambda$ are positive constants to be determined. We then have

$$
H(\eta) \sim A^{p} \eta^{\lambda p-2} \quad \text { as } \eta \rightarrow \infty .
$$

Since $M[H ; z]$ has a simple pole

$$
M[H ; z] \sim-\frac{A^{p}}{z-(2-\lambda p)} \quad \text { as } z \rightarrow 2-\lambda p,
$$

if $\lambda p>1$ and the vertical path of integration in the complex $z$-plane lies between the two poles, the leading asymptotic contribution of the integral in (3.6) comes from the Mellin transform. Equation (3.6) takes the form

$$
A \eta^{\lambda} \sim \frac{A^{p} \eta^{\lambda p-1}}{\lambda p-1} \quad \text { as } \eta \rightarrow \infty .
$$

Matching the two sides of (3.11) then yields

$$
\lambda=\frac{1}{p-1} \quad \text { and } \quad A=\left(\frac{1}{p-1}\right)^{\frac{1}{p-1}} .
$$

We next let $h(w) \sim e^{w}$ and assume that

$$
w(\eta) \sim \log (A \eta) \sim \log \eta+\log A \quad \text { as } \eta \rightarrow \infty .
$$

We then have

$$
H(\eta) \sim A \eta^{-1} \quad \text { as } \eta \rightarrow \infty .
$$

Thus, $M[H ; z] \sim-\frac{A}{z-1}$ as $z \rightarrow 1$. To compute the leading asymptotic contribution from the integral in (3.6), the vertical path is displaced to the right. In this case, $z=1$ is a double pole, and hence (3.6) takes the form

$$
\log \eta \sim A \log \eta \quad \text { as } \eta \rightarrow \infty
$$

which gives $A=1$.

In summary, we have the following.

If $h(u) \sim u^{p}$, then $u(0, t) \sim\left(\frac{1}{p-1}\right)^{\frac{1}{p-1}}(\hat{t}-t)^{-\frac{1}{p-1}}$ as $t \rightarrow \hat{t}$;

If $h(u) \sim e^{u}$, then $u(0, t) \sim \log \left(\frac{1}{\hat{t}-t}\right)$ as $t \rightarrow \hat{t}$. 


\section{REFERENCES}

[1] N. Bleistein and R. A. Handelsman, Asymptotic Expansion of Integrals, Holt, Rinehart and Winston, New York, 1975

[2] C. Corduneanu, Integral Equations and Applications, Cambridge University Press, New York, 1991

[3] V. A. Galaktionov and H. A. Levine, On critical Fujita exponents for heat equations with nonlinear flux conditions on the boundary, Israel J. Math. 94, 125-146 (1996)

[4] R. T. Glassey, Finite-time blow-up for solutions of nonlinear wave equations, Math. Z. 177, 323-340 (1981)

[5] T. Kato, Blow-up of solutions of some nonlinear hyperbolic equations, Comm. Pure Appl. Math. 32, 501-505 (1980)

[6] W. E. Olmstead and C. A. Roberts, Explosion in a diffusive strip due to a concentrated nonlinear source, Methods Math. Anal. 1, 434-445 (1994)

[7] C. A. Roberts and W. E. Olmstead, Growth rates for blow-up solutions of nonlinear Volterra equations, Quart. Appl. Math. 54, 153-160 (1996)

[8] T. Sideris, Nonexistence of global solutions to semilinear wave equations in high dimensions, J. Differential Equations 52, 378-406 (1984) 